\title{
Improvements in tongue strength and pressure-generation precision following a tongue-pressure training protocol in older individuals with dysphagia: Three case reports
}

\author{
Erin M Yeates' \\ Sonja M Molfenter' \\ Catriona M Steele $\mathrm{e}^{1,2,3,4}$ \\ 'Toronto Rehabilitation Institute, \\ Toronto, Canada; ${ }^{2}$ Department \\ of Speech-Language Pathology, \\ University of Toronto, Toronto, \\ Canada; ${ }^{3}$ Canadian Institutes \\ of Health Research New Investigator \\ in Aging; ${ }^{4}$ Bloorview Kids Rehab, \\ Toronto, Canada
}

\begin{abstract}
Dysphagia, or difficulty swallowing, often occurs secondary to conditions such as stroke, head injury or progressive disease, many of which increase in frequency with advancing age. Sarcopenia, the gradual loss of muscle bulk and strength, can place older individuals at greater risk for dysphagia. Data are reported for three older participants in a pilot trial of a tongue-pressure training therapy. During the experimental therapy protocol, participants performed isometric strength exercises for the tongue as well as tongue pressure accuracy tasks. Biofeedback was provided using the Iowa Oral Performance Instrument (IOPI), an instrument that measures tongue pressure. Treatment outcome measures show increased isometric tongue strength, improved tongue pressure generation accuracy, improved bolus control on videofluoroscopy, and improved functional dietary intake by mouth. These preliminary results indicate that, for these three adults with dysphagia, tongue-pressure training was beneficial for improving both instrumental and functional aspects of swallowing. The experimental treatment protocol holds promise as a rehabilitative tool for various dysphagia populations.
\end{abstract}

Keywords: speech-language pathology, dysphagia, rehabilitation, aging, strength, accuracy

\section{Introduction}

As the North American population ages, the health care system is becoming increasingly aware of geriatric health concerns and strategies for their management. One such issue in the elderly is swallowing difficulty (dysphagia). Estimates of dysphagia prevalence in the United States range from $40 \%$ to $50 \%$ of adults aged 60 and older (Robbins et al 2005; Chen et al 2008). As many as $80 \%$ of elderly individuals residing in skilled nursing facilities have been reported to exhibit observable clinical signs of swallowing difficulty at the mealtime (Steele et al 1997).

Dysphagia is a component of many different disease and injury processes, including acute neurological events, chronic progressive neurological disease, and structural lesions of the head and neck. Oropharyngeal dysphagia (difficulties in the oral or pharyngeal phases of swallowing) can manifest with a variety of features, including reduced efficiency and effectiveness of tongue movements for bolus propulsion and transfer, difficulty initiating the pharyngeal swallowing response, and inadequate or mistimed airway protection. People with dysphagia are at risk for life-threatening sequelae such as dehydration, malnutrition, and airway obstruction (Finestone et al 1995, 1996, 1998; Palmer et al 2000). In particular, they are at risk for developing lung infections such as pneumonitis or pneumonia secondary to aspiration (the invasion of foreign material into the airway) (Pikus et al 2003). Dysphagia may be short-lived and resolve, but many individuals experience permanent difficulty with 
swallowing. These individuals may require artificial nutrition and hydration or may be subject to dietary restrictions and texture modification for the long-term. Furthermore, swallowing difficulties frequently bring about anxiety around eating and withdrawal in social settings (Ekberg et al 2002; Bennett and Steele 2005; Chen et al 2008).

Several studies in the literature concur that age-related changes occur in the swallowing mechanism. It has been reported that individuals over the age of 65 , on average, swallow more slowly than people under the age of 45 (Sonies et al 1984; Tracy et al 1989; Sonies 1992; Robbins et al 1992; Logemann et al 2000). Other reported age-related changes include: increased lingual connective tissue resulting in reduced masticatory strength and a longer oral phase (Jaredah 1994); extra lingual movements during bolus formation (Caruso and Max 1997); reduced lingual pressure (Shaker and Lang 1994); increased pharyngeal residue following the swallow (Tracy et al 1989; Robbins et al 1992); and reduced coordination of the oral and pharyngeal phases of swallowing (Tracy et al 1989). Furthermore, as lingual driving force is reduced with age, there is an increased likelihood of bolus retention in the valleculae (Dejaeger et al 1997), putting the elderly patient at risk of post-swallow aspiration.

The geriatric population is reported to be at particular risk for swallowing difficulties due to age-related changes in the head and neck musculature (Robbins et al 2005). Sarcopenia is a progressive loss of skeletal muscle mass and strength associated with aging (Evans 1995). Sarcopenia in the larger striated muscle groups has been implicated as the cause of functional decline and decompensation and is suspected in smaller striated muscles, such as those found in the head and neck (Robbins et al 2005). The tongue is composed entirely of muscle and plays a critical role in swallowing by generating propulsive forces for transporting food and liquid through the oropharynx and into the esophagus. Reduced functional muscle reserve in the tongue and orofacial musculature is thought to heighten vulnerability to physiologically distressing states in elderly people (Robbins et al 2005). As the population ages, dysphagia and its consequences are being more frequently associated with such common age-related conditions as congestive heart failure, diabetes mellitus, and frailty (Kendall et al 2004; Robbins et al 2005). Conceivably, age-related reductions in muscle mass and strength could exacerbate the effects of neurogenic or structural changes to the swallowing mechanism.

Recent studies show that healthy adults exhibit agerelated decline in functional motor capacity of the tongue, but that tongue pressure generation exercises can restore muscle mass and strength (Robbins et al 2005). During swallowing, the tongue applies pressure to the bolus by rising towards the palate and generating an anterior-to-posterior pressure wave that squeezes liquids backwards towards the pharynx (Chi-Fishman et al 1998; Hiiemae and Palmer 2003). We hypothesized that the ability to control the flow of thin liquids in the oral phase of swallowing requires the ability to modulate the amplitude of pressure applied to the bolus by the tongue. Swallowing pressures are known to be considerably smaller in amplitude than maximum isometric pressure capacity (Nicosia et al 2000). We therefore developed a tongue pressure training protocol that emphasized both strength (ie, maximum pressures) and accuracy (ie, the ability to generate submaximal pressures to match specific targets). To our knowledge, tongue pressure accuracy has not previously been studied in healthy or disordered individuals. In this manuscript, we report treatment progress and outcomes in a preliminary case report of three adults with dysphagia involving tongue weakness and difficulty with liquid bolus control. The results for these three participants involved are descriptive and preliminary, and cannot be generalized as a group.

\section{Methods}

Tongue pressure generation exercises were conducted with biofeedback from the Iowa Oral Performance Instrument (IOPI; Blaise Medical Inc., Hendersonville, TN, USA). The IOPI registers tongue-palate pressures via a single airfilled bulb, approximately $2 \mathrm{~cm} \times 1 \mathrm{~cm} \times 0.5 \mathrm{~cm}$. This bulb connects into a device that shows the recorded pressures (in kilopascals) on a numeric LCD display. The bulb is held in the mouth in either an anterior or posterior position. When completing exercises in the anterior position, the participant uses the tongue tip to press the bulb towards the centre of the alveolar ridge, just behind the front teeth. Exercises in the posterior position involve pressing the bulb with the midpoint of the tongue to the midpoint of the hard palate.

The experimental treatment protocol involved the execution of ten sets of 6 reiterated tongue-to-palate presses per session, half in the anterior and half in the posterior bulb position. Half of the exercises were isometric strength exercises, for which the participant was instructed to press the bulb to the roof of their mouth as hard as possible. In cases where the participant was able to generate a volitional saliva swallow, one set of 6 tongue presses was performed in the anterior position, coupled with a swallow. The remaining exercises were accuracy tasks, in which the participant was instructed to generate precise pressures in either the anterior or posterior 
bulb location. The target amplitudes for this accuracy task were set by the speech-language pathologist at 50\%, 75\%, and $100 \%$ of the maximum pressure measured during the first isometric strength exercise in the session for each bulb location. Success in performing each task was communicated directly to the participant following each attempt, by reporting the pressure value registered on the IOPI device. Patients were seen for therapy 2-3 times per week for 45-minute sessions with a speech-language pathologist. There was no home practice component outside of the therapy sessions with the speech-language pathologist.

\section{Participants}

Data are reported for three participants who were selected to participate based on demonstrated difficulties with oral control for thin liquids on their videofluoroscopic swallowing studies (VFSS). Posterior spillover, reduced base of tongue movement and vallecular residue were used as gross measures of difficulties with oral control to qualify participants for the study.

\section{Case A}

Case A was a 72-year-old male, who at the initiation of therapy was 7 months post-stroke. The location of the stroke was in the left lateral medullary region. At the onset of treatment, he was receiving approximately half of his daily nutrition by gastrostomy tube supplemented by a soft texture oral diet with thickened fluids. He received 2-3 therapy sessions per week and completed 24 therapy sessions, with pre-treatment, midway, and post-treatment VFSS assessments.

\section{Case B}

Case B was a 63-year-old male, who at the initiation of therapy was 42 months post-IVth ventricle tumor resection. The surgery resulted in damage to cranial nerves XI (spinal accessory) and XII (hypoglossal) as well as left vocal cord paresis. The patient had no history of radiation or chemotherapy for treatment of the tumor. At the onset of treatment, he was receiving primary nutrition by gastrostomy tube supplemented by soft texture oral snacks, sips of water by teaspoon and thickened fluids. He was seen 3 times per week for swallowing therapy and completed 24 sessions, with pretreatment, midway, and post-treatment VFSS assessments.

\section{Case C}

Case $\mathrm{C}$ was a 50-year-old male, who at the initiation of therapy was 34 months post-motor vehicle accident that resulted in a brainstem stroke. At the onset of treatment, he was receiving full nutritional support by gastrostomy tube and taking no food or liquid by mouth. The treatment schedule for this patient differed from the other two, due to the fact that he lived in another city. After 8 sessions of intensive daily face-to-face therapy and training in the therapy protocol, this patient returned to his home and continued home practice with an IOPI 3 times per week for 90 sessions. He recorded his pressure measurements on a tracking sheet provided to him by the speech-language pathologist, and reported all measurements to the clinician within 12 hours by email. The speechlanguage pathologist, in turn, reviewed the daily results and sent new accuracy targets for the subsequent session by return email. Therapy is currently ongoing. This participant has received VFSS at baseline and at two points midway through therapy, after 41 sessions and after 66 sessions.

\section{Analysis}

We monitored three tongue-palate pressure outcome measures during this experimental therapy study: (a) average peak pressures measured in anterior and posterior positions on the isometric strength tasks; (b) accuracy (ie, rectified distance from the specified target) on the accuracy tasks; and (c) accuracy relative to strength, (ie, rectified distance from the specified target on the accuracy tasks, divided by the maximum isometric pressure measured during the strength tasks). All measures were calculated separately for the anterior and posterior pressure bulb positions. These measures are shown for each individual participant in Figures 1-14. Physiologic changes were measured at the pre-, midway, and post-treatment intervals using VFSS, a dynamic video X-ray of the oropharynx, which is considered the gold standard in dysphagia assessment. Specifically, from the VFSS recordings, we calculated stage transition duration time (STDT) as a measure representing bolus control. STDT is defined as the interval between: (a) the arrival of the head of the bolus at the lower margin of the shadow of the ramus of mandible and, (b) the onset of the first hyolaryngeal excursion associated with upper esophageal (UES) opening (Power et al 2007). Hyolaryngeal excursion usually precedes the arrival of thin liquid boluses in the pharynx in healthy people. In the event of poor bolus control in people with dysphagia, the bolus arrives prematurely in the pharynx and the onset of the swallow can be significantly delayed (Power et al 2007).

\section{Individual results}

\section{Case A}

Case A began his treatment with isometric strength values that were close to the normative range reported for healthy, 


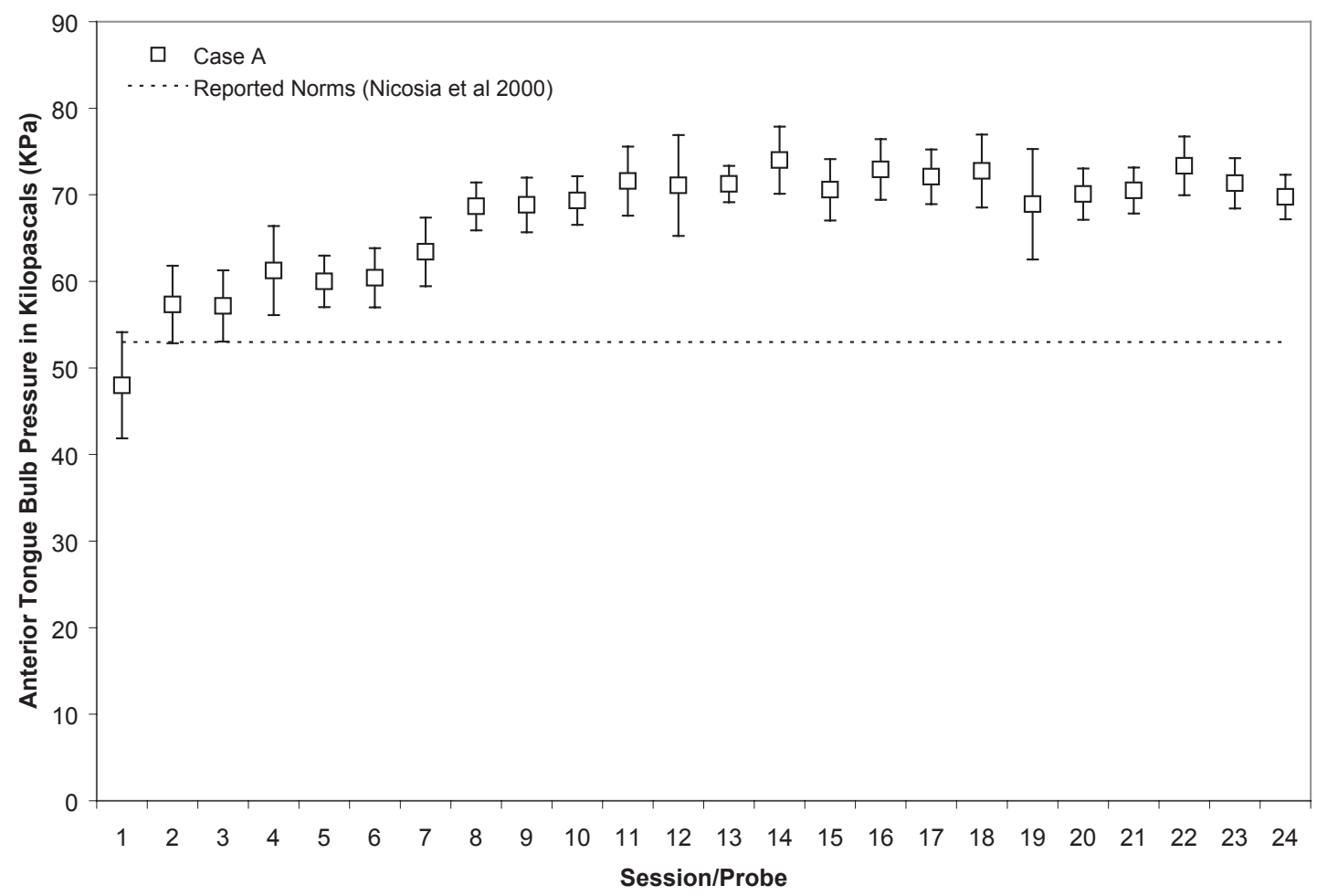

Figure I Means (with standard deviations shown by error bars) for maximum isometric tongue pressure measurements (in kilopascals), collected with the IOPI bulb in the anterior position, are shown across the course of treatment for Case A. The dotted line shows, for reference, the normative values for maximum anterior isometric tongue pressure generation in healthy older males reported by Nicosia and colleagues (2000).

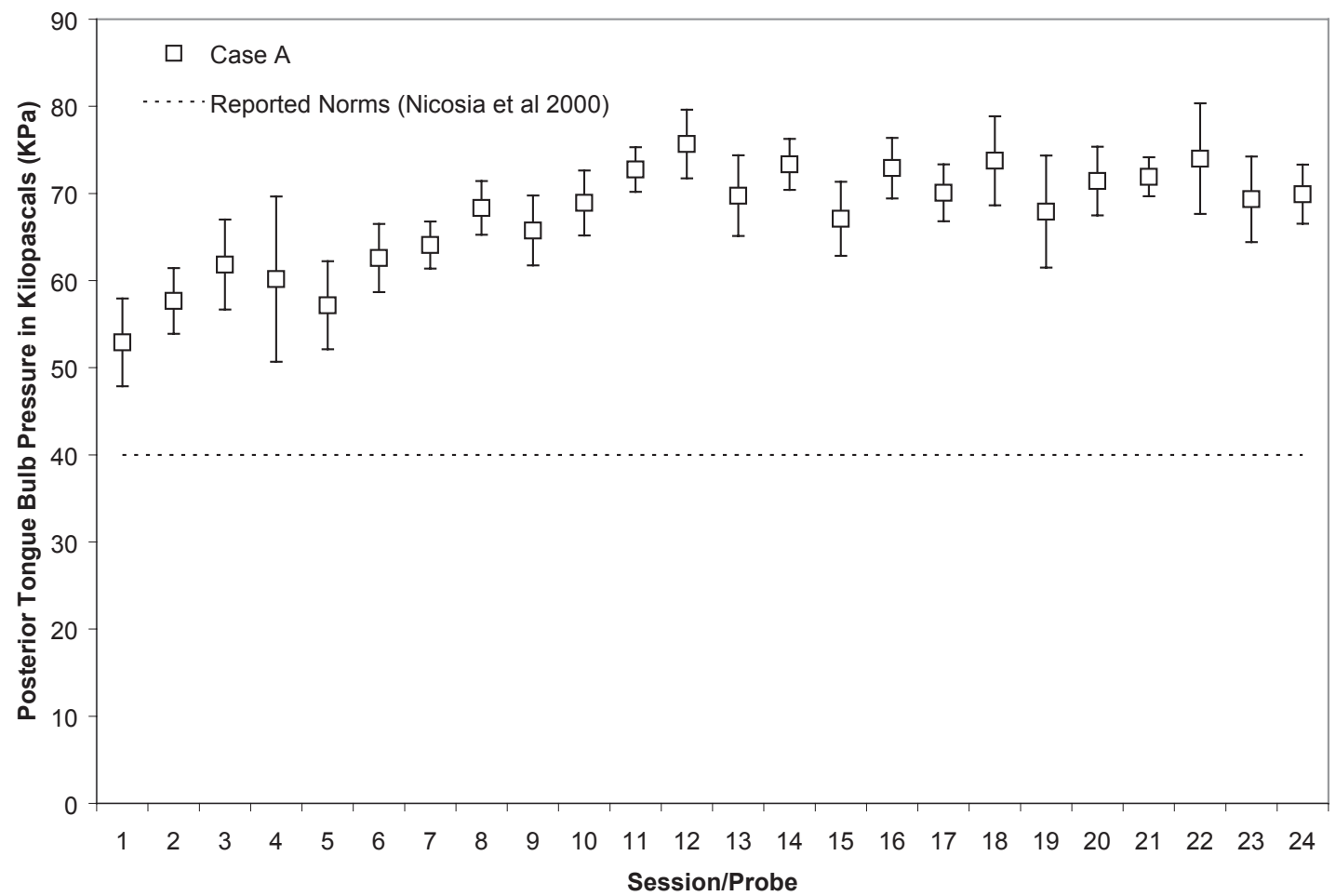

Figure 2 Means (with standard deviations shown by error bars) for maximum isometric tongue pressure measurements (in kilopascals), collected with the IOPI bulb in the posterior position, are shown across the course of treatment for Case A. The dotted line shows, for reference, the normative values for maximum posterior isometric tongue pressure generation in healthy older males reported by Nicosia and colleagues (2000). 


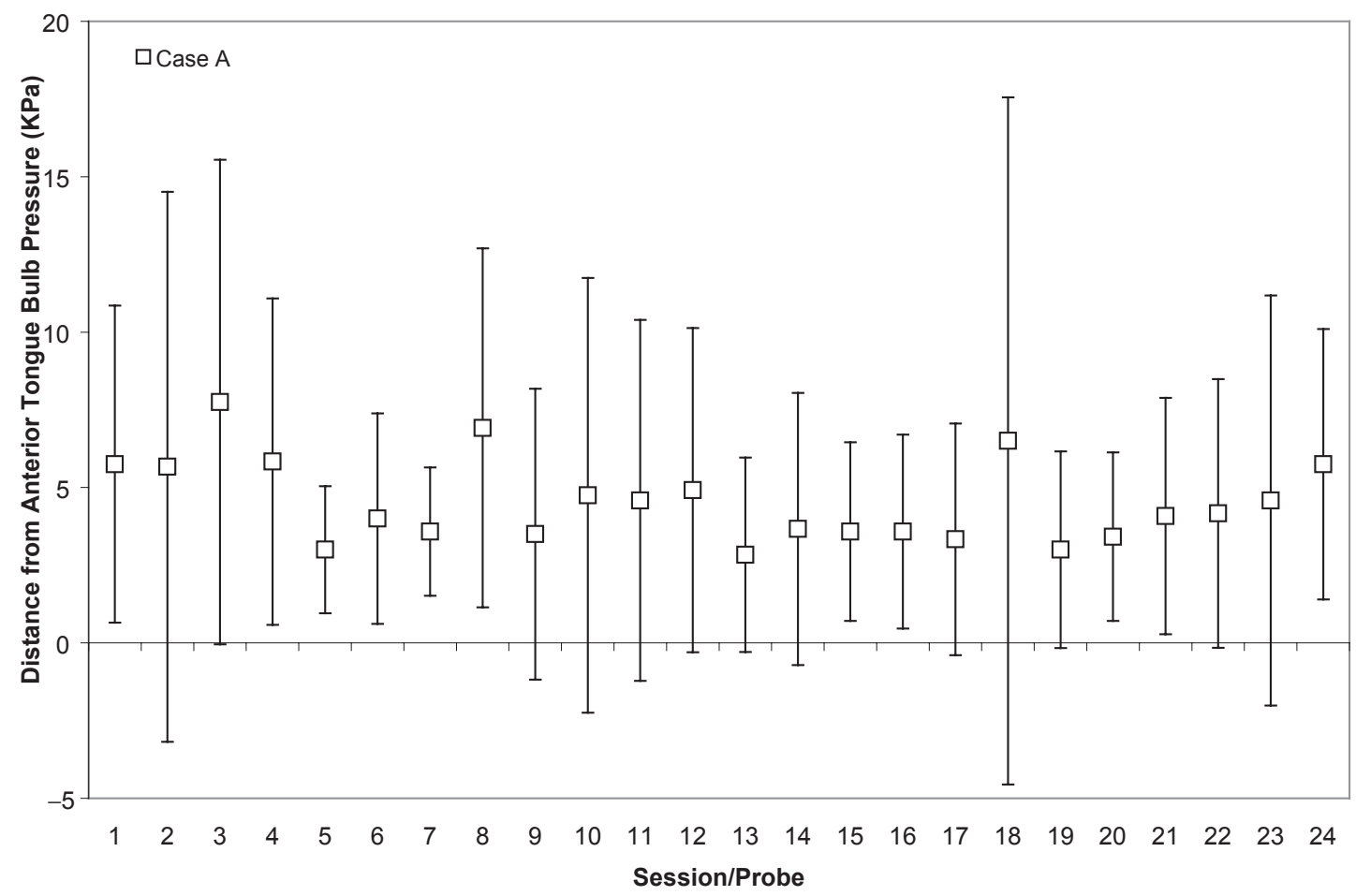

Figure 3 Means (with standard deviations shown by error bars) for tongue pressure accuracy (ie, distance from a specified target in kilopascals), collected with the IOPI bulb in the anterior position, are shown across the course of treatment for Case $A$.

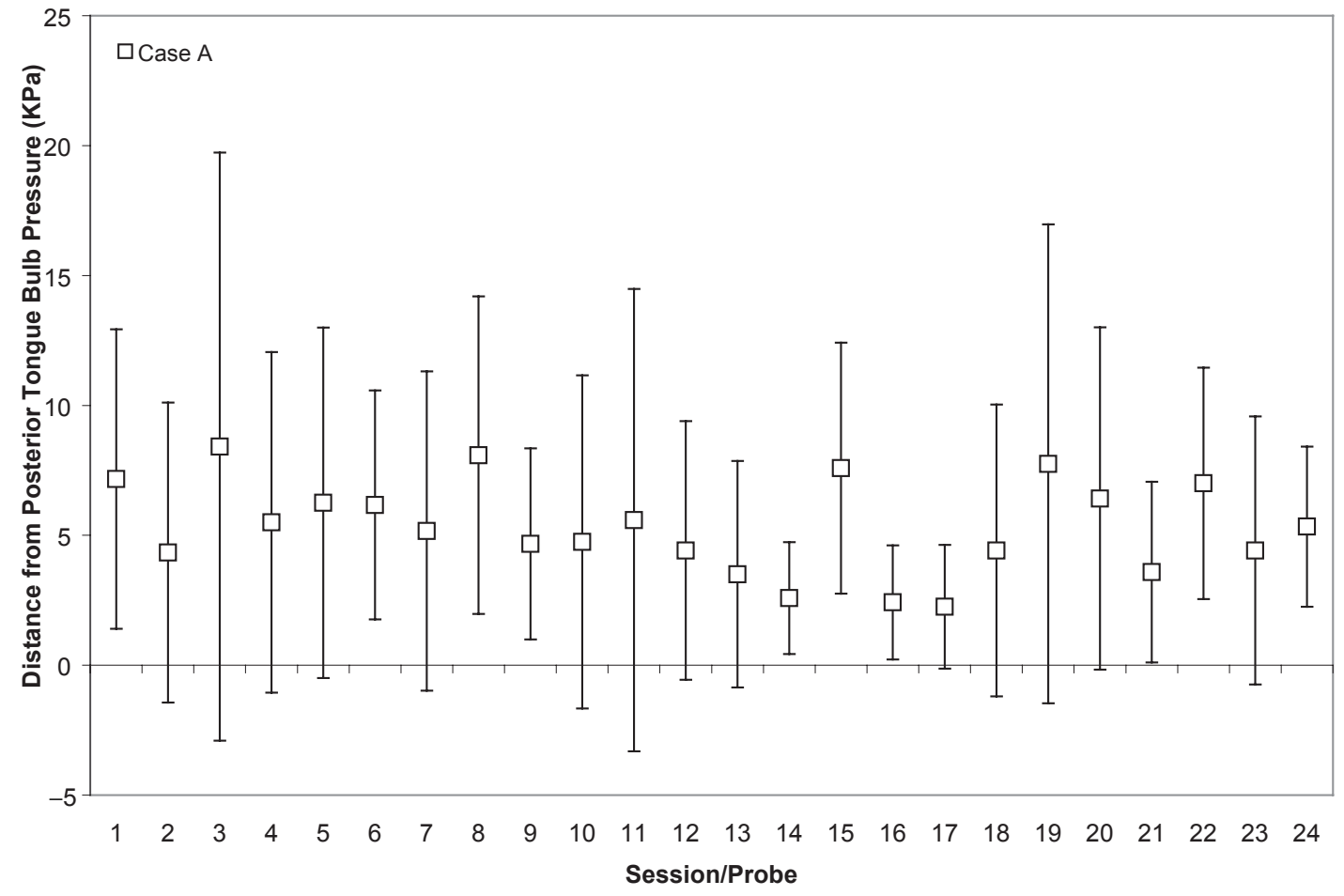

Figure 4 Means (with standard deviations shown by error bars) for tongue pressure accuracy (ie, distance from a specified target in kilopascals), collected with the IOPI bulb in the anterior position, are shown across the course of treatment for Case A. 


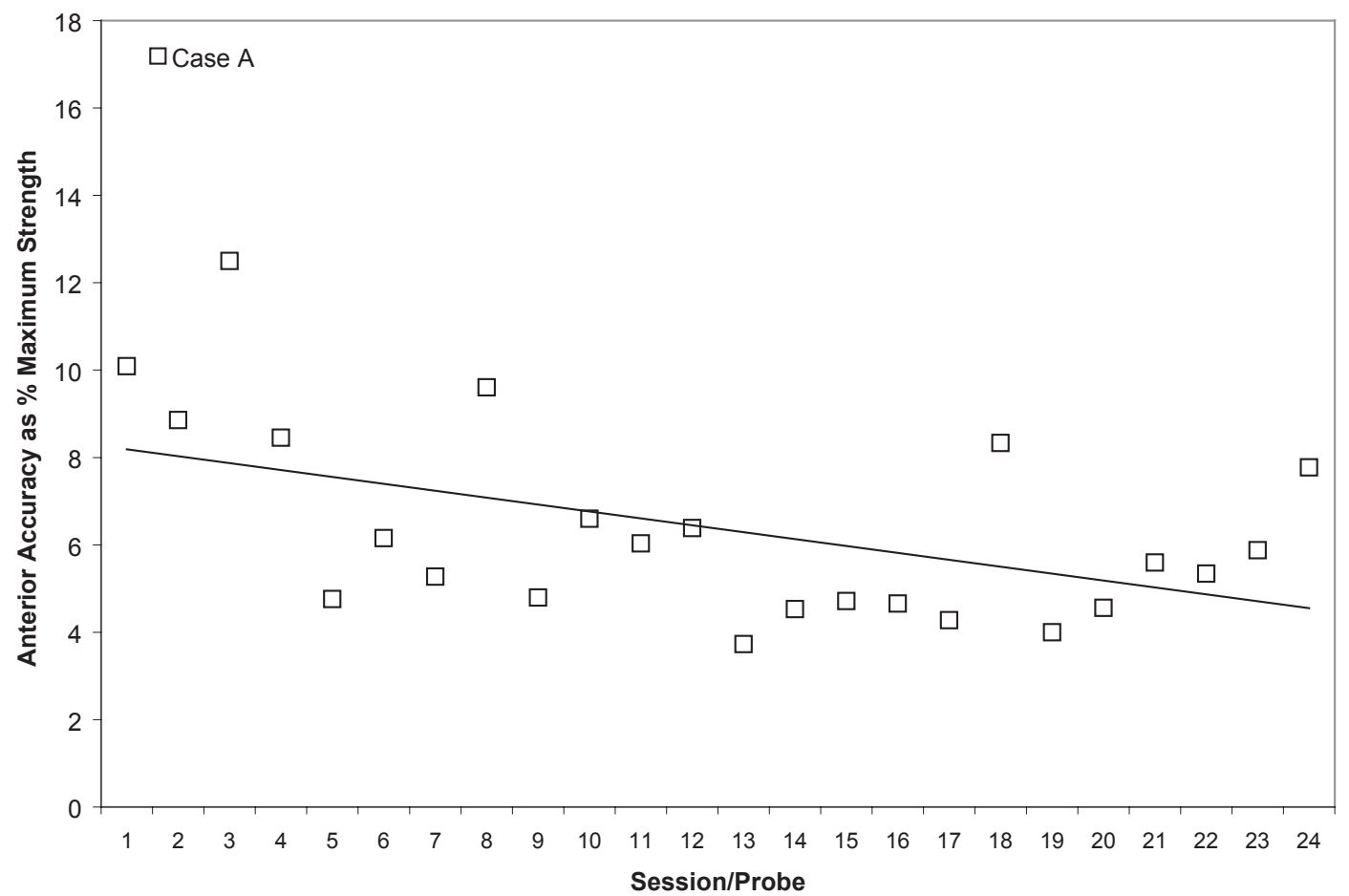

Figure 5 Mean anterior tongue pressure accuracy (ie, distance from a specified target, measured in kilopascals), is shown as a percentage of the maximum isometric anterior tongue pressure measured (in kilopascals) in the corresponding treatment session for Case A. The linear trend line shows the pattern of change across the course of treatment.

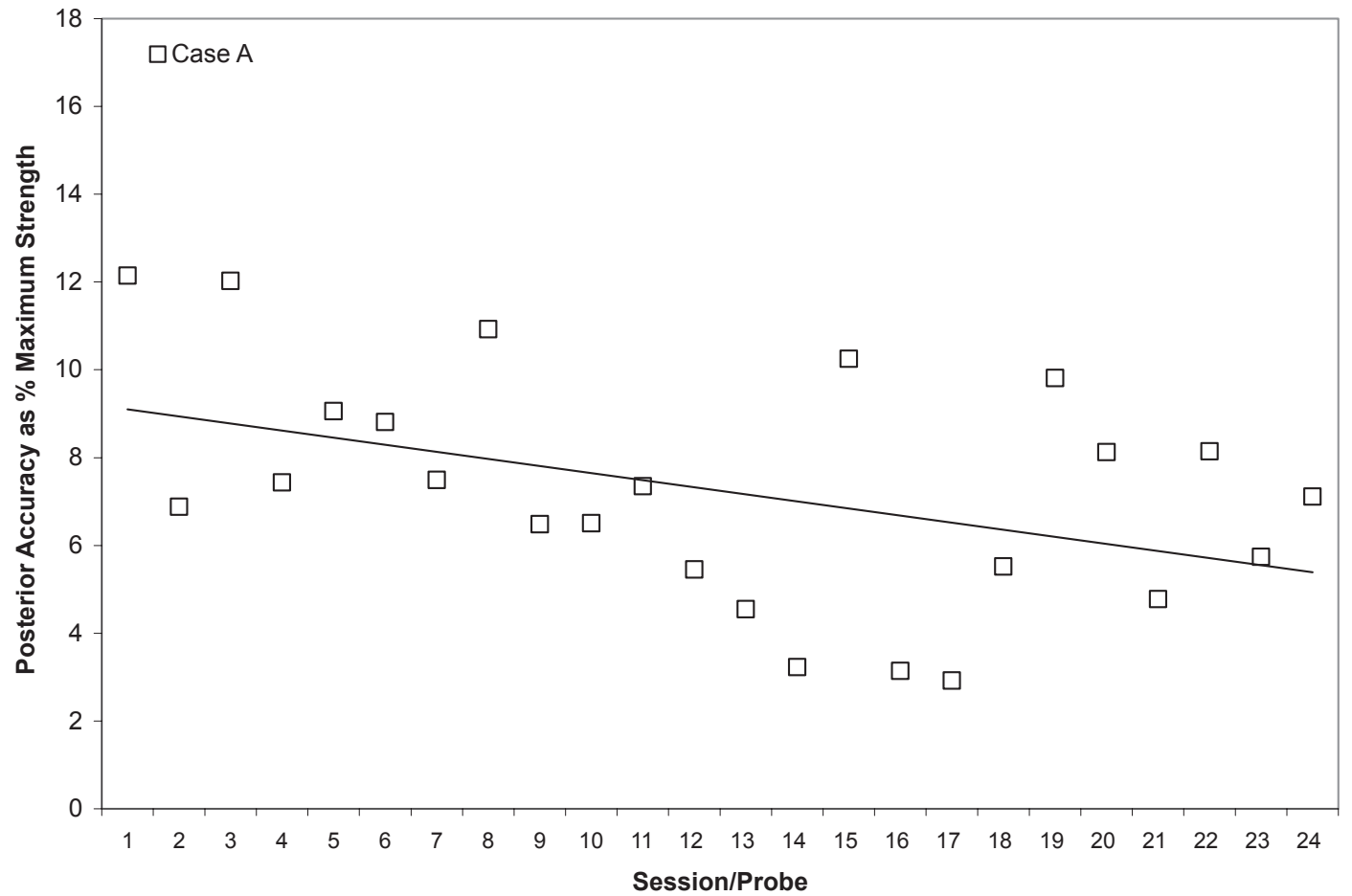

Figure 6 Mean posterior tongue pressure accuracy (ie, distance from a specified target, measured in kilopascals), is shown as a percentage of the maximum isometric posterior tongue pressure measured (in kilopascals) in the corresponding treatment session for Case A. The linear trend line shows the pattern of change across the course of treatment. 


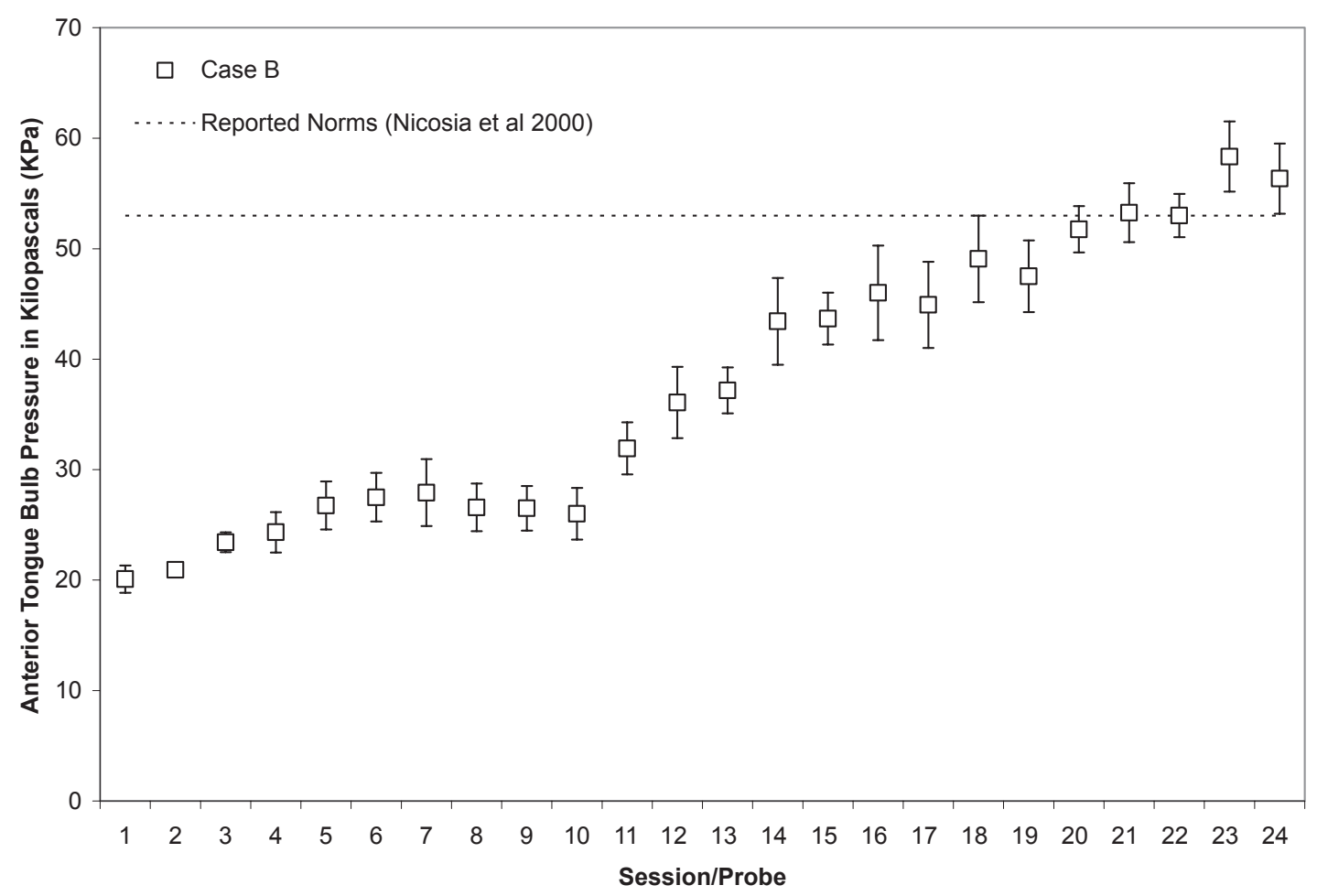

Figure 7 Means (with standard deviations shown by error bars) for maximum isometric tongue pressure measurements (in kilopascals), collected with the IOPI bulb in the anterior position, are shown across the course of treatment for Case B. The dotted line shows, for reference, the normative values for maximum anterior isometric tongue pressure generation in healthy older males reported by Nicosia and colleagues (2000).

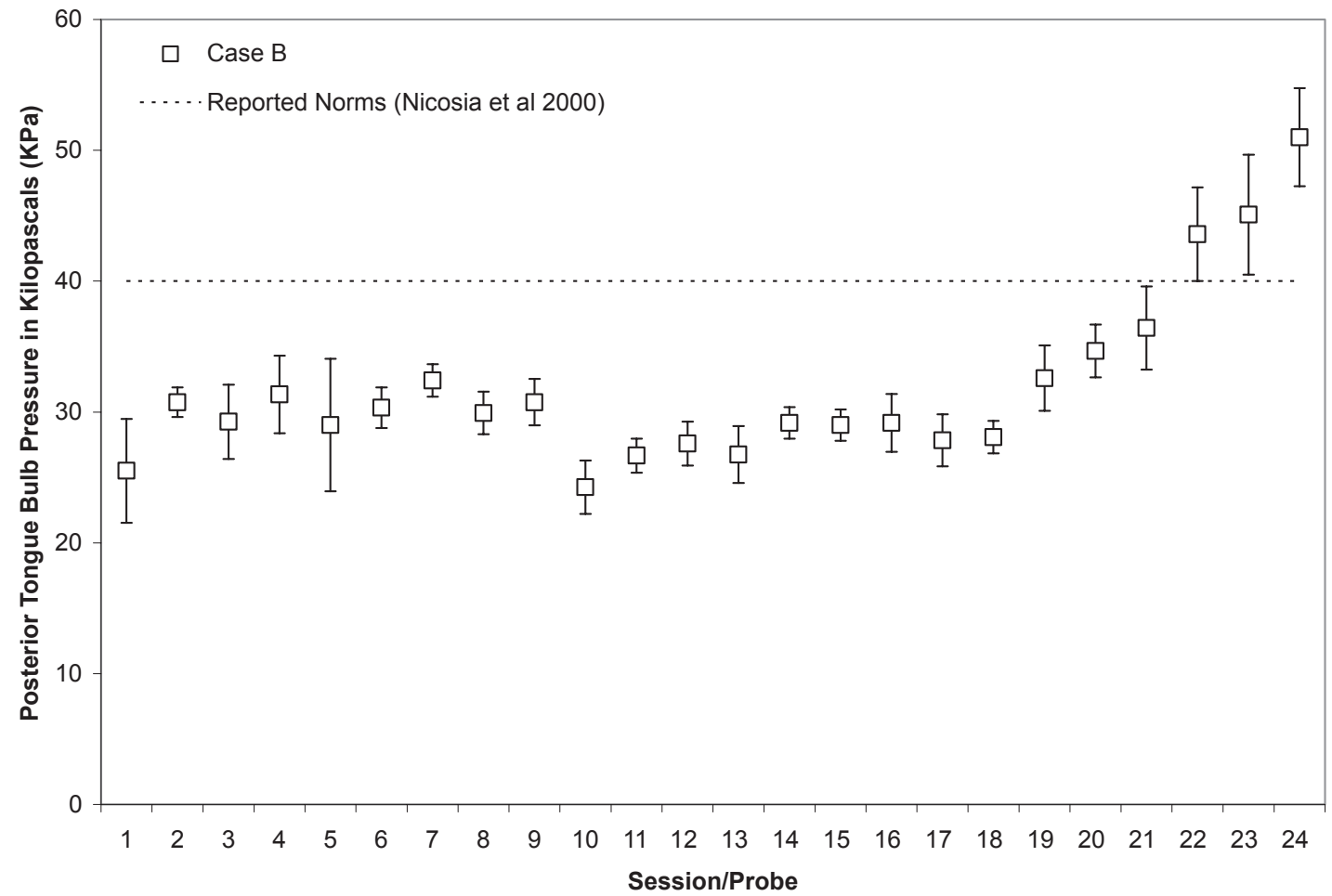

Figure 8 Means (with standard deviations shown by error bars) for maximum isometric tongue pressure measurements (in kilopascals), collected with the IOPI bulb in the posterior position, are shown across the course of treatment for Case B. The dotted line shows, for reference, the normative values for maximum posterior isometric tongue pressure generation in healthy older males reported by Nicosia and colleauges (2000). 


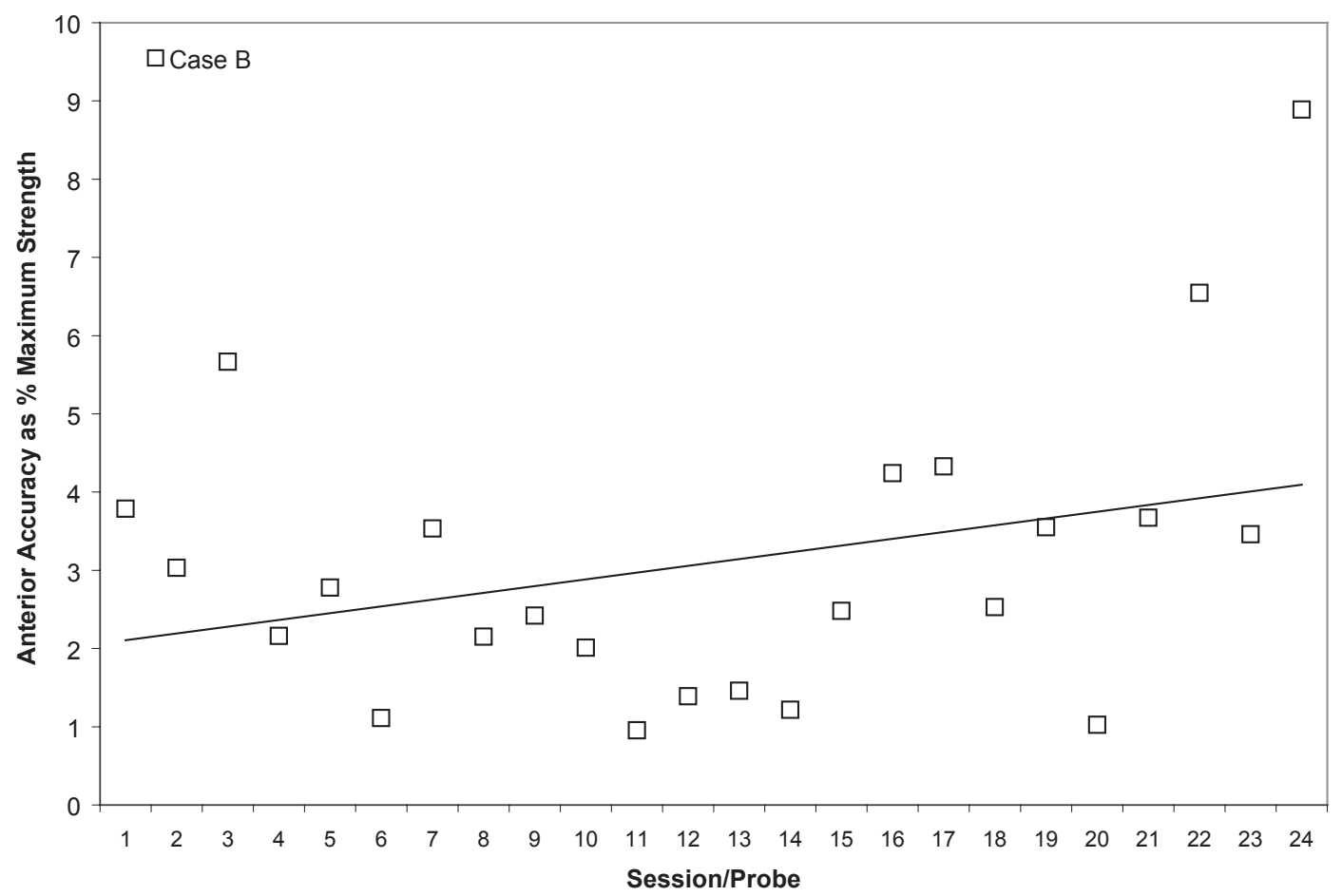

Figure 9 Mean anterior tongue pressure accuracy (ie, distance from a specified target, measured in kilopascals), is shown as a percentage of the maximum isometric anterior tongue pressure measured (in kilopascals) in the corresponding treatment session for Case B. The linear trend line shows the pattern of change across the course of treatment.

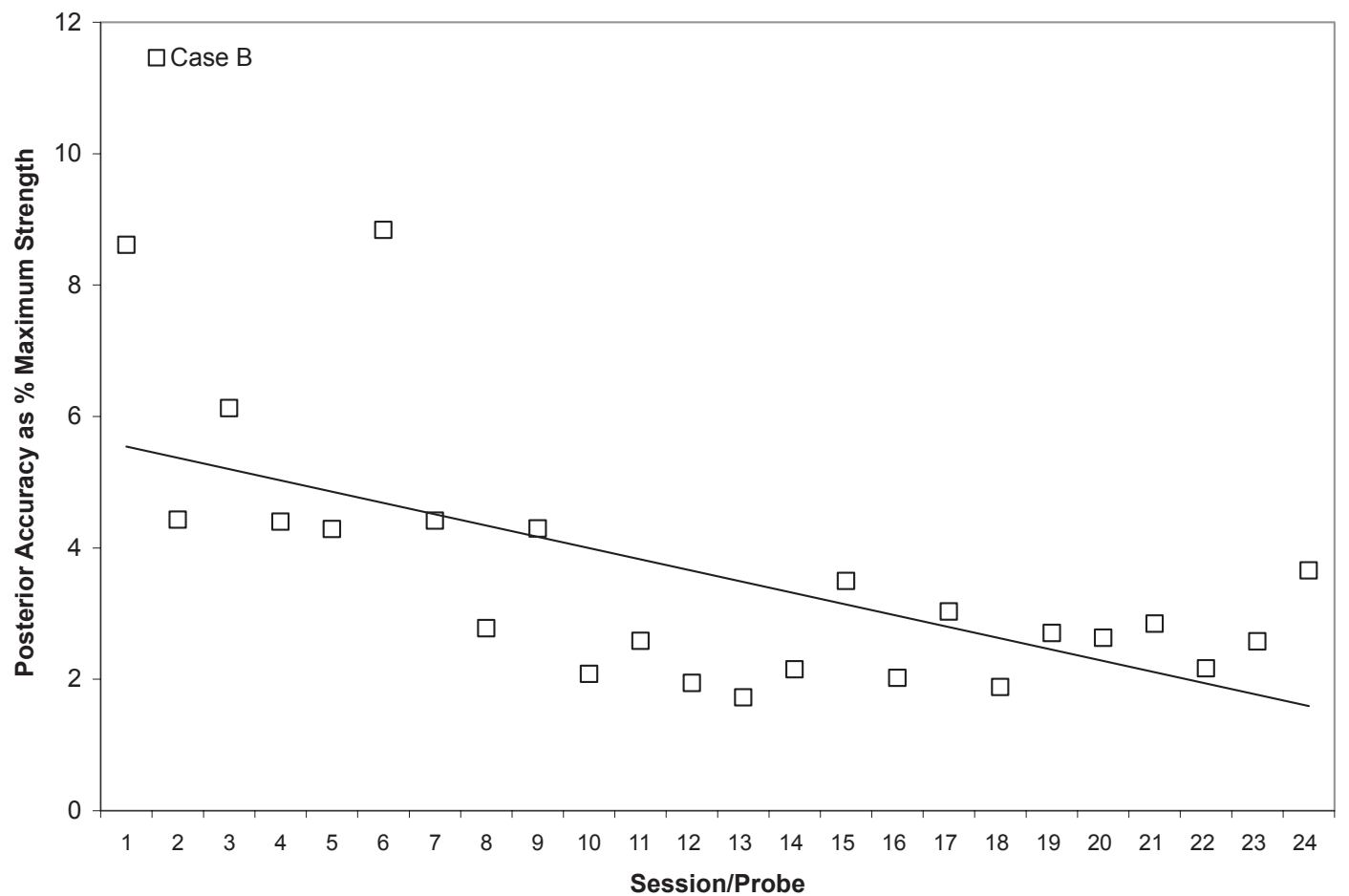

Figure 10 Mean posterior tongue pressure accuracy (ie, distance from a specified target, measured in kilopascals), is shown as a percentage of the maximum isometric posterior tongue pressure measured (in kilopascals) in the corresponding treatment session for Case B. The linear trend line shows the pattern of change across the course of treatment. 


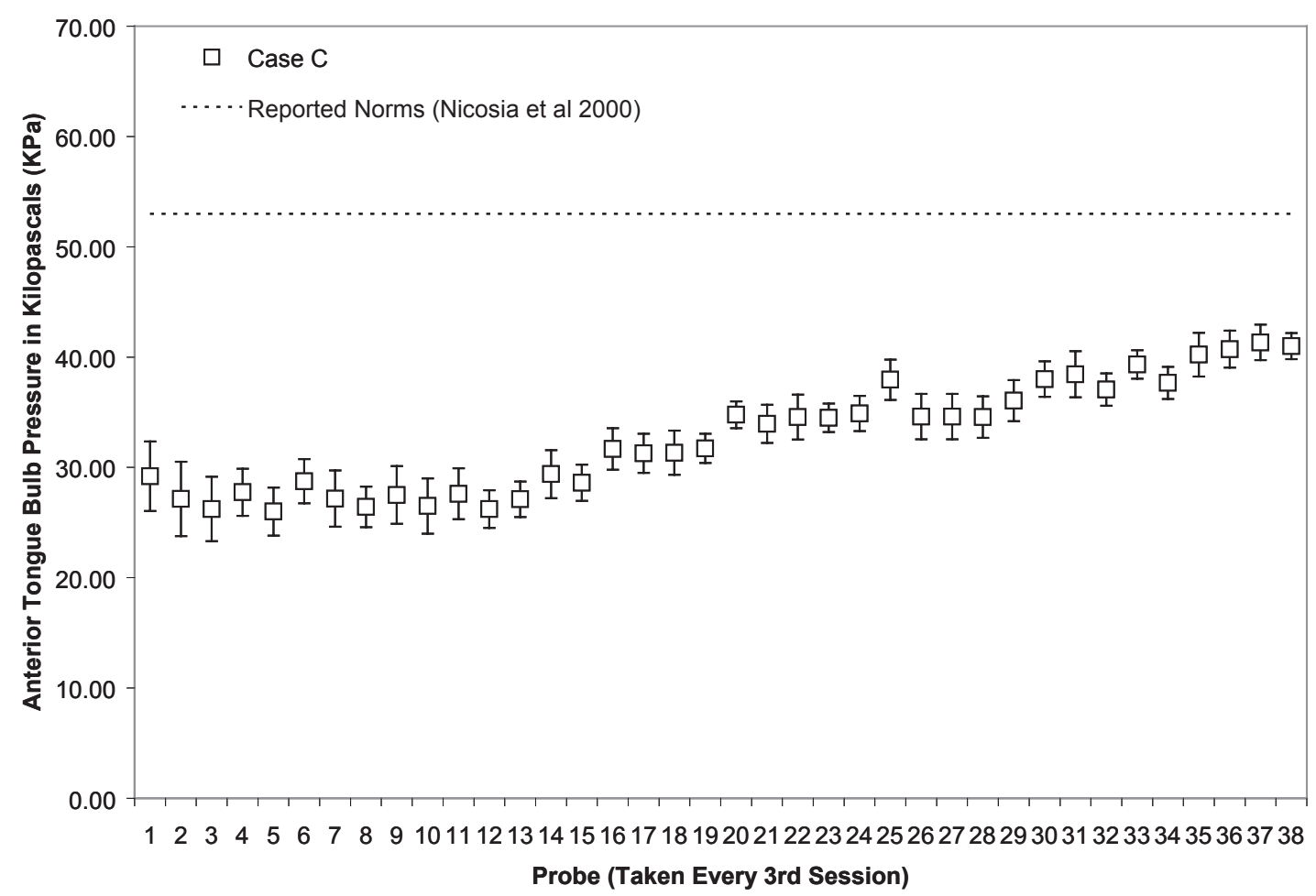

Figure II Means (with standard deviations shown by error bars) for maximum isometric tongue pressure measurements (in kilopascals), collected with the IOPI bulb in the anterior position, are shown across the course of treatment for Case C. The dotted line shows, for reference, the normative values for maximum anterior isometric tongue pressure generation in healthy older males reported by Nicosia and colleagues (2000).

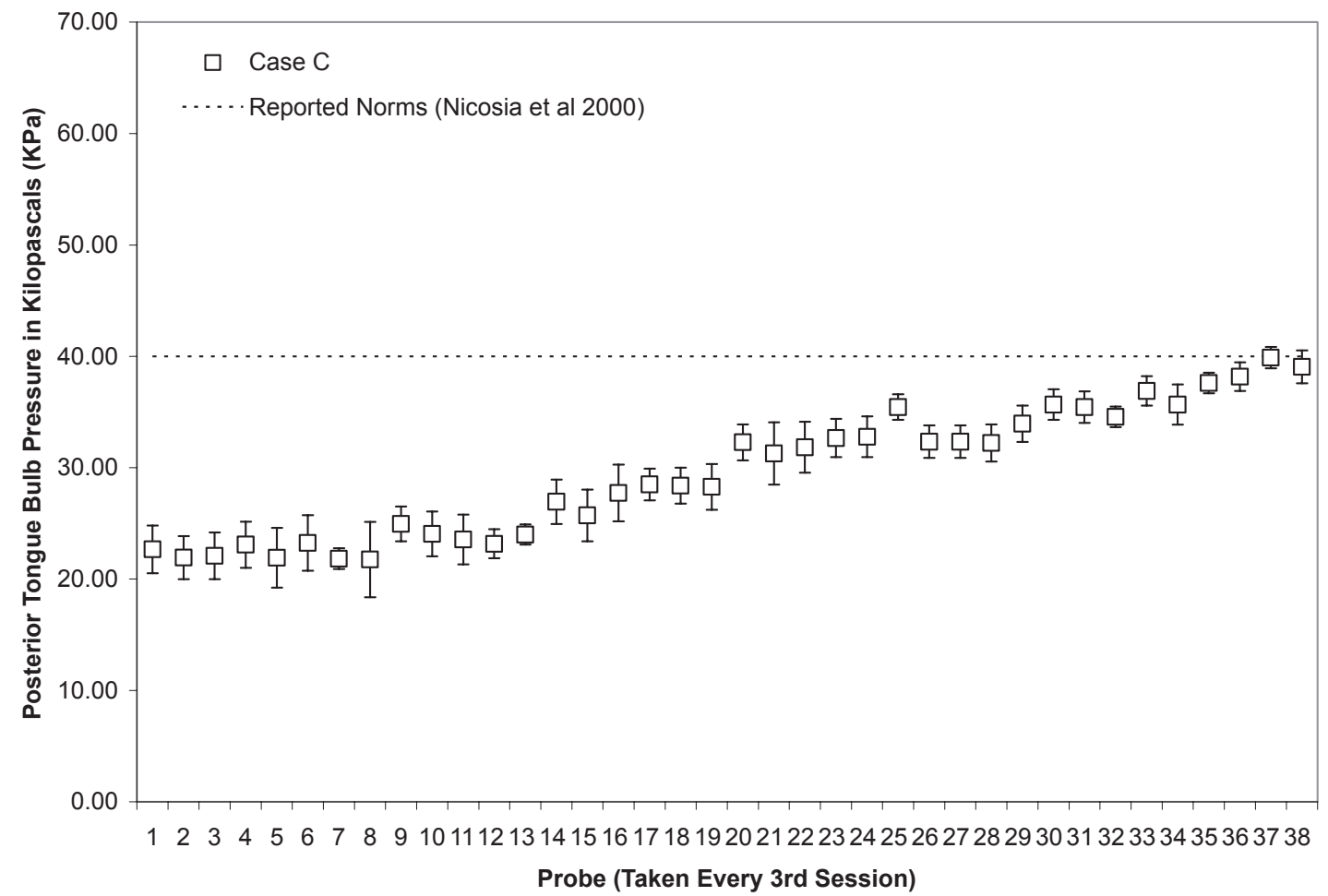

Figure 12 Means (with standard deviations shown by error bars) for maximum isometric tongue pressure measurements (in kilopascals), collected with the IOPI bulb in the posterior position, are shown across the course of treatment for Case B.The dotted line shows, for reference, the normative values for maximum posterior isometric tongue pressure generation in healthy older males reported by Nicosia and colleagues (2000). 


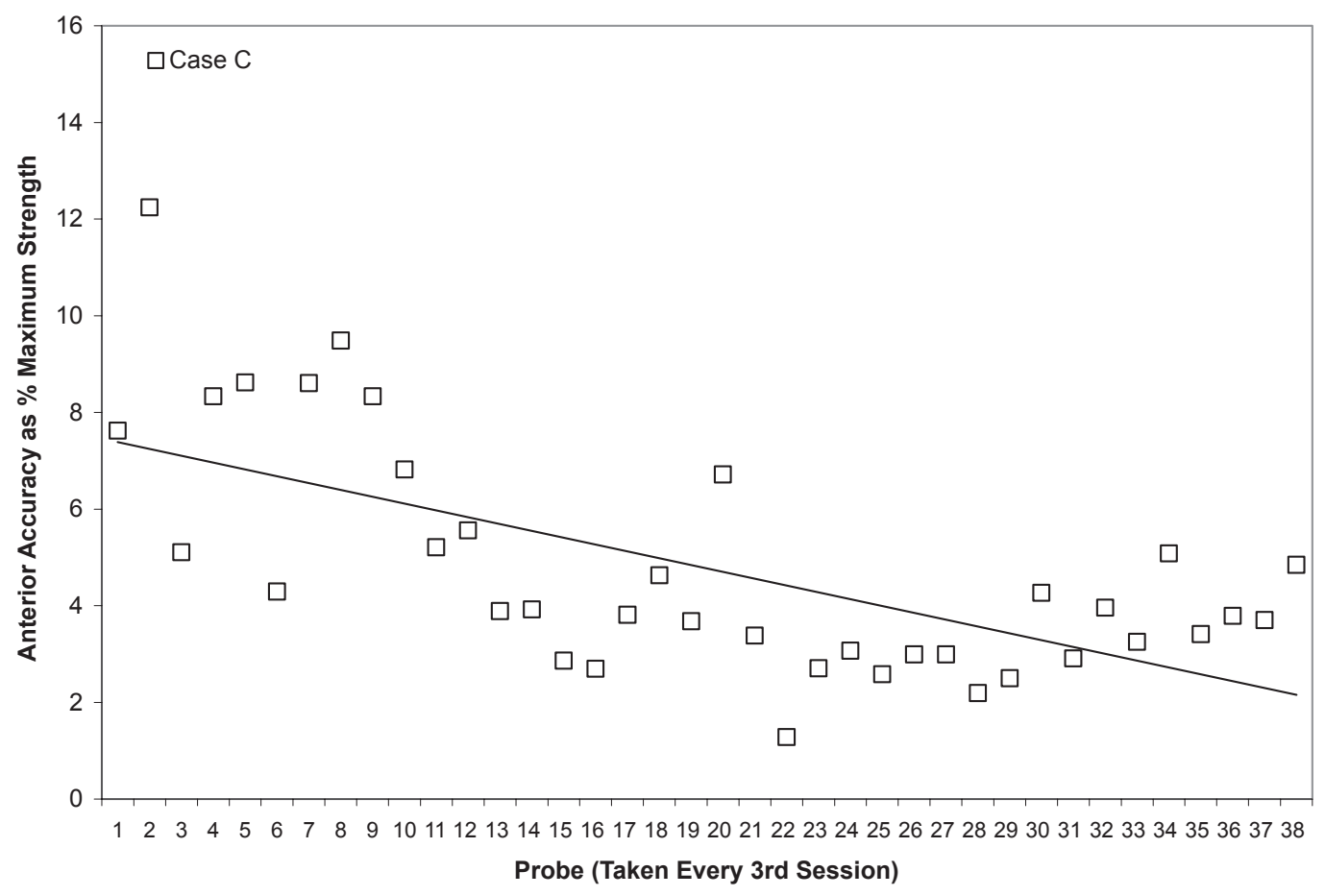

Figure 13 Mean anterior tongue pressure accuracy (ie, distance from a specified target, measured in kilopascals), is shown as a percentage of the maximum isometric anterior tongue pressure measured (in kilopascals) in the corresponding treatment session for Case C. The linear trend line shows the pattern of change across the course of treatment.

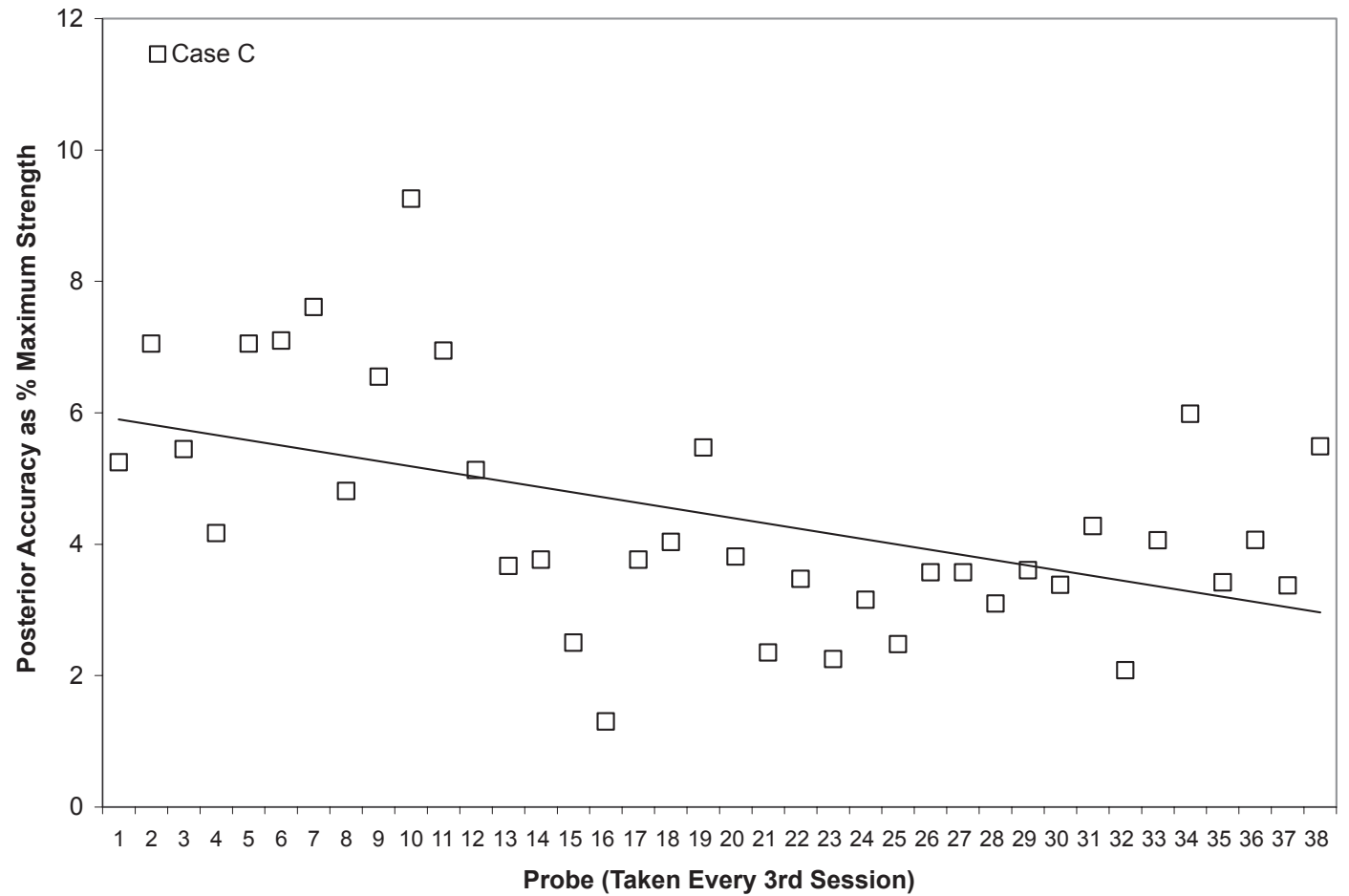

Figure 14 Mean posterior tongue pressure accuracy (ie, distance from a specified target, measured in kilopascals), is shown as a percentage of the maximum isometric posterior tongue pressure measured (in kilopascals) in the corresponding treatment session for Case $\mathrm{C}$. The linear trend line shows the pattern of change across the course of treatment. 
older males for the anterior bulb position (Nicosia et al 2000), namely $48 \pm 6 \mathrm{KPa}$, and within the normative reported range for the posterior bulb position, ie, $53 \pm 5 \mathrm{KPa}$. His isometric pressure measures increased rapidly, achieving a $50 \%$ increase versus baseline by the 11th session for the anterior bulb position and a $38 \%$ increase for the posterior bulb position in the same time frame. These changes are illustrated in Figures 1 and 2, for which the mean and standard deviations of each session's isometric pressure measurements are represented by the white data points and error bars, and the reported normative value from the literature is shown by the dotted line on the chart.

Case A's ability to achieve accuracy targets was volatile across most of his therapy course. He began with absolute values between 5 and $10 \mathrm{kPa}$ from target for both bulbs. Although he improved slightly by the end of his treatment to produce pressures on accuracy tasks with mean values within $5 \mathrm{kPa}$ of target no particular trend is appreciable from plots of these measures across the course of therapy (Figures 3 and 4). However, recognizing that accuracy might vary in part as a function of strength, we also chose to examine the mean accuracy measures for each session in a relative sense, as a percentage of the isometric strength values collected in the corresponding session. This representation can be seen in Figures 5 and 6 for Case A, with a trend line showing an overall downward pattern, ie, an improvement in tongue pressure accuracy relative to strength, for both bulb positions over the course of treatment. With respect to physiologic change, Case A's mean stage transition duration time decreased from $830 \mathrm{~ms}$ pre-treatment to $100 \mathrm{~ms}$ post-treatment. Functional outcomes included increased oral intake to the extent that tube feeding was discontinued, and tolerance for an increased variety of soft foods and for thin fluids. By the end of treatment, this participant was taking his medication orally.

\section{Case B}

As shown in Figures 7 and 8, Case B began his treatment with isometric pressure measures well below the reported norms for both the anterior and posterior bulb locations. Furthermore, up until the midpoint of therapy, Case B showed little change in these isometric strength measures. However, strength measures then began to increase gradually in the anterior position, ultimately reaching the normative range (Nicosia et al 2000). Case B's ability to reach accuracy targets was outstanding from the outset. Figure 9 illustrates an increase in volatility for anterior pressure accuracy as his strength measures improved, whereas his accuracy for the posterior bulb location improved across the course of therapy (Figure 10). In VFSS, Case B's mean stage transition duration time decreased from $2220 \mathrm{~ms}$ pre-treatment to $560 \mathrm{~ms}$ post-treatment. Functional outcomes included an increase in the amount and variety of oral intake tolerated. Over the course of treatment, Case B gradually decreased the number of cans of enteral nutrition he ingested by gastrostomy tube per day. Feeding by gastrostomy tube continued for partial nutritional support, but oral intake steadily increased over the course of therapy.

\section{Case C}

Due to the large number of sessions completed by Case $C$, data points were plotted for every 3rd session across the entire course of observation (including both the initial face to face sessions and the subsequent home practice sessions). As shown in Figures 11 and 12, this patient showed the slowest change in average isometric pressures. Small, but steady and gradual increases were observed, with a net change of approximately $10 \mathrm{kPa}$ in peak values for the anterior bulb position and $20 \mathrm{kPa}$ for the posterior bulb position. Accuracy, represented relative to isometric strength, showed a trend towards improvement for both bulb positions across the course of treatment (Figures 13 and 14). Stage transition duration could not be determined reliably due to the absence of UES opening. However, on the most recent outcome videofluoroscopy, after session 66, transient opening of the UES was observed for the first time since the patient's injury. At this time, functional swallowing has not returned, and NPO status and enteral nutrition remain in place.

\section{Discussion}

These results illustrate different patterns of response to therapy in three patients with dysphagia of differing etiology and chronicity. The most acute patient, Case A, was the most similar to other cases reported in the literature, both in terms of chronicity and the relative gains in strength that were achieved (Robbins et al 2007). This patient had the largest and steadiest increases in isometric strength, and was able to achieve strength levels considered appropriate for his age (Nicosia et al 2000). Paired with his significant gains in both anterior and posterior strength, he displayed improvements in accuracy measures across the course of therapy. Case B showed no significant changes in strength until midway through his treatment. As his strength improved, he showed a deterioration in accuracy for the anterior tongue, but accuracy for the posterior tongue improved across the course of therapy. Case $\mathrm{C}$, who had the most severe dysphagia 
(including inability to achieve UES opening), took an even greater amount of time to show increased strength. His accuracy measures have shown continuous improvement in both positions across the duration of his treatment. With the exception of Case B's anterior values, all three participants showed downward trends (ie, improvement) on accuracy tasks across the course of therapy. Although this is a preliminary sample of data from a small group of subjects, this finding suggests that accuracy training, in addition to strength training, may also contribute to the rehabilitation of swallowing difficulties.

\section{Future research directions}

Previous research has shown beneficial effects of tongue strengthening therapy in older adults (Robbins 2005) and in adults with primarily acute-phase stroke (Robbins 2007). The preliminary, descriptive results described here indicate that patients with chronic, longer-standing swallowing difficulties may require longer periods of time to demonstrate positive changes in strength and accuracy measures, as well as functional swallowing outcomes. However, improvements in tongue strength and accuracy appear to be possible even at these late stages. This is important information, particularly for elderly individuals, who might have longer-standing swallowing problems resulting from multiple etiologies. It will be necessary to study a large and varied group of older patients with chronic dysphagia to expand the new perspective gleaned from the present research.

Interestingly, in these three cases, accuracy appears to improve over the course of therapy, and may be an important component of a lingual pressure training program. In this protocol, we measured accuracy in terms of pressure amplitude. However, it should be noted that bolus control may also require temporal precision in tongue pressure generation. Consequently, it is recommended that future studies should examine both the magnitude and time course of tongue pressure generation during both maximum effort and precision tasks.

Finally, these descriptive results hold preliminary significance for the field of swallowing rehabilitation. Dysphagia is increasingly recognized as a disorder secondary to a variety of etiologies that increase in prevalence with age. Therefore, as the population ages exponentially, so too will the number of people living with complex chronic diseases and dysphagia. The ability of clinicians to rehabilitate this common health issue will be essential for the future quality of care for the ever-expanding aging population.

\section{Acknowledgments}

This research was supported by funding from the Canadian Institutes of Health Research (MOP-84534, SIC-83888). Equipment and space have been funded with grants from the Canada Foundation for Innovation and the Province of Ontario. The authors acknowledge the support of Toronto Rehabilitation Institute who receives funding under the Provincial Rehabilitation Research Program from the Ministry of Health and Long-term Care in Ontario. The views expressed do not necessarily reflect those of the ministry.

\section{References}

Bennett JW, Steele CM. 2005. The impact of dysphagia on quality of life. Perspectives on Swallowing and Swallowing Disorders (Dysphagia), 14:24-7.

Caruso AJ, Max L. 1997. Effects of aging on neuromotor processes of swallowing. Semin Speech Lang, 18:181-92.

Chen PH, Golub JS, Hapner ER, et al. 2008. Prevalence of perceived dysphagia and quality-of-life impairment in a geriatric population. Dysphagia, Mar 27. [Epub ahead of print].

Chi-Fishman G, Stone M, McCall GN. 1998. Lingual action in normal sequential swallowing. J Speech Lang Hear Res, 41:771-85.

Dejaeger E, Pelemans W, Ponette E, et al. 1997. Mechanisms involved in postdeglutition retention in the elderly. Dysphagia, 12:63-7.

Ekberg O, Hamdy S, Woisard V, et al. 2002. Social and psychological burden of dysphagia: its impact on diagnosis and treatment. Dysphagia, $17: 139-46$.

Evans WJ. 1995. What is sarcopenia? J Gerontol A Biol Sci Med Sci, $50: 5-9$.

Finestone HM, Fisher J, Greene-Finestone LS, et al. 1998. Sudden death in the dysphagic stroke patient - a case of airway obstruction caused by a food bolus: a brief report. Am J Phys Med Rehabil, 77:550-2.

Finestone HM, Greene-Finestone LS, Wilson ES, et al. 1995. Malnutrition in stroke patients on the rehabilitation service and at follow-up: prevalence and predictors. Arch Phys Med Rehabil, 76:310-6.

Finestone HM, Greene-Finestone LS, Wilson ES, et al. 1996. Prolonged length of stay and reduced functional improvement rate in malnourished stroke rehabilitation patients. Arch Phys Med Rehabil, 77:340-5.

Hiiemae KM, Palmer JB. 2003. Tongue movements in feeding and speech. Crit Rev Oral Biol Med, 14:413-29.

Jaredah S. 1994. Neurophysiology of swallowing in the aged. Dysphagia, 9:218-20.

Kendall KA, Leonard RJ, McKenzie S. 2004. Common medical conditions in the elderly: impact on pharyngeal bolus transit. Dysphagia, 19:71-7.

Logemann JA, Pauloski BR, Rademaker AW, et al. 2000. Temporal and biomechanical characteristics of oropharyngeal swallow in younger and older men. J Speech Lang Hear Res, 43:1264-74.

Nicosia MA, Hind JA, Roecker EB, et al. 2000. Age effects on the temporal evolution of isometric and swallowing pressure. J Gerontol A Biol Sci Med Sci, 55:M634-40.

Palmer JB, Drennan JC, Baba M. 2000. Evaluation and treatment of swallowing impairments. Am Fam Physician, 61:2453-62.

Pikus L, Levine MS, Yang YX, et al. 2003. Videofluoroscopic studies of swallowing dysfunction and the relative risk of pneumonia. AJR Am J Roentgenol, 180:1613-6.

Power ML, Hamdy S, Singh S, et al. 2007. Deglutitive laryngeal closure in stroke patients. J Neurol Neurosurg Psychiatry, 78:141-6.

Robbins J, Gangnon RE, Theis SM, et al. 2005. The effects of lingual exercise on swallowing in older adults. $J$ Am Geriatr Soc, 53:1483-9.

Robbins J, Hamilton JW, Lof GL, et al. 1992 Oropharyngeal swallowing in normal adults of different ages. Gastroenterology, 103:823-9. 
Robbins J, Kays SA, Gangnon RE, et al. 2007. The effects of lingual exercise in stroke patients with dysphagia. Arch Phys Med Rehabil, 88:150-8.

Shaker R, Lang IM. 1994. Effect of aging on the deglutitive oral, pharyngeal and esophageal motor function. Dysphagia, 9:221-8.

Sonies BC. 1992. Oropharyngeal dysphagia in the elderly. Clin Geriatr Med, 8:569-77.
Sonies BC, Stone M, Shawker T. 1984. Speech and swallowing in the elderly. Gerodontology, 3:115-23.

Steele CM, Greenwood C, Ens I, et al. 1997. Mealtime difficulties in a home for the aged: not just dysphagia. Dysphagia, 12:43-50.

Tracy JF, Logemann JA, Kahrilas PJ, et al. 1989. Preliminary observations on the effects of age on oropharyngeal deglutition. Dysphagia, 4:90-4. 
